\title{
Extraction of Zernike Coefficients Modes using Holographic Wavefront Sensing: Modeling and Simulation
}

\author{
S. Kara Mohammed, D. Bahloul, S. Cherif, and M. Rahmani
}

\begin{abstract}
We propose a holographic wavefront sensor that uses a binary computer-generated hologram (BCGH). This CGH is coded with a single particular Zernike aberration mode with different coeffecients and then all the holograms are multiplexed and binarized to get the final hologram. This multiplexed hologram is used to produce a spots (white pixel on the detector) according to the presence of particular aberration. The Numerical results by Matlab program corresponding to the CGHs are presented.
\end{abstract}

Index Terms-Zernike aberration modes, wavefront sensing, Fourier transform, computer-generated hologram.

\section{INTRODUCTION}

As an important part of the adaptive optics system, wavefront sensor can detect the unknown wavefront aberrations coming through various sources e.g., optical component, atmosphere, etc. In modal wavefront sensors the aberration can be decomposed into a set of orthogonal polynomials (e.g. Zernike polynomials [1], or Zernike aberration mode). If only the coefficient of each Zernike aberration mode is fixed, we will retrieve the aberration in formation and fully reconstruct the distorted wavefront. Various techniques exist in the literature for wavefront sensing [2]-[4], each with their own benefits and drawbacks. Recently, a holographic wavefront sensor has been proposed [5], [6], in which a multiplexed hologram acts as a wavefront sensor. It completely removes the need of any computation. However, optical recording of multiplexed hologram is tedious process.

The computer-generated hologram is the better option to implement this type of sensor [7], [8].

Our work is about the development of a holographic wavefront sensor to be used for ophtalmic applications such as in vivo retinal imaging and human eye's aberrations measurements.

Manuscript received May 20, 2015; revised July 27, 2015

S. Kara Mohammed is with the Centre for Development of Advanced Technologies (CDTA), Research Unit in Optics and Photonics (UROP), Instrumentation and Modern Optics Research team, Farhat Abbas University1, El Bez, 19000 Setif, Algeria and Applied Optics Laboratory, Institute of Optics and Precision Mechanics, Ferhat Abbas University of Setif, 19000 Setif, Algeria (e-mail: skaramohammed@cdta.dz).

D. Bahloul, S. Cherif, and M. Rahmani are with the Department of Material Science, Faculty of Sciences, Hadj Lakhdar University, Batna, Algeria and Centre for Development of Advanced Technologies (CDTA), Optics and Photonics Research Unit (UROP), Instrumentation and Modern Optics Research team, Farhat Abbas University1, El Bez, Setif-19000, Algeria (e-mail: dbahloul@hotmail.com, scherif@cdta.dz, mrahmani@cdta.dz).

\section{COMPUTER GENERATED HOLOGRAM}

We record each CGH by interfering a reference wave $\Psi_{R}^{k}=$ $\Psi_{p} \exp \left(i \frac{2 \pi}{\lambda} a_{k} Z_{k}\right)$ with an object wave $\psi_{O}^{k} . \Psi_{R}^{k}$ is the reference wave distorted by a Zernike aberration mode $\varphi_{k}=a_{k} Z_{k}$ with $\mathrm{k}$ the single index. $\psi_{O}^{k}$ is the object wave passing through an image with white pixel and an FT lens. The record process is represented in Fig. 1.

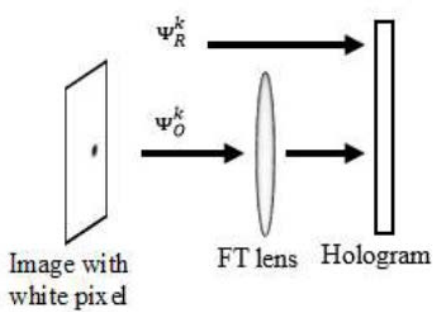

Fig. 1. Recording process for a single hologram.

According to the principle of holography [9], the intensity at the recording plane of the single hologram is calculated by:

$$
\begin{gathered}
I_{k}=\left[\psi_{a}^{k}+F T(d p)\left[\psi_{a}^{k}+F T(d p)\right]^{*}\right. \\
I_{k}=\left|\psi_{a}^{k}\right|^{2}+\left|F T\left(d_{p}\right)\right|^{2}+\psi_{a}^{k}\left[F T\left(d_{p}\right)\right]^{*}+\left[\psi_{a}^{k}\right]^{*} F T\left(d_{p}\right) \\
I_{k}=\left|\psi_{p} \exp \left(i \frac{2 \pi}{\lambda} a_{k} Z_{k}\right)\right|^{2}+\left|F T\left(d_{p}\right)\right|^{2}+ \\
\psi_{p} \exp \left(i \frac{2 \pi}{\lambda} a_{k} Z_{k}\right)\left[F T\left(d_{p}\right)\right]^{*}+\left[\psi_{p} \exp \left(i \frac{2 \pi}{\lambda}\right)\right]^{*} F T\left(d_{p}\right)
\end{gathered}
$$

For the amplitude hologram, its transmission $\tau$ can be viewed as proportional to the interferogram intensity distribution $I_{k}$. We choose the aberrated wave $\Psi_{a}^{k}$ as the replay wave to reconstruct the hologram, and multiply the four terms on right hand side of (3) equation using $\Psi_{a}^{k}$. In the result, first two terms are zero diffraction order, third term reconstructs the conjugate object beam, the fourth term gives the original object beam, and if we take its inverse Fourier transform, we retrieve the image with white pixel. Fig. 2 gives the reconstruction process.

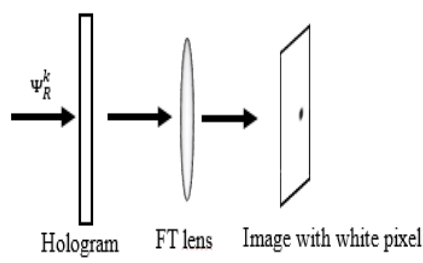

Fig. 2. Reconstruction process for a single hologram. 
The corresponding spot in the detector plane represent the existence of the aberration $a_{k} Z_{k}$; therefore, we can achieve the goal to detect the unknown aberration quickly and effectively.

\section{NUMERICAL SimUlATION AND RESUlts}

In order to show the interest of holographic wavefront sensor, CGH for the coma1 aberration with different coefficients has been simulated by applying equation (3). The associated parameters in numerical simulations are set as follows: wavelength $632.8 \mathrm{~nm}$, pixel number $256 \times 256$, coefficients values (from $-2 \lambda$ to $2 \lambda$ ) without the zero value, which is not required to design the hologram. The obtained interference pattern between a plane wavefront and the aberrated wavefront is as shown in Fig. 1.

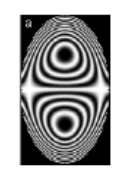

$-2 \lambda$

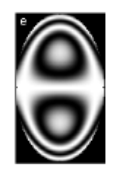

$0.5 \lambda$

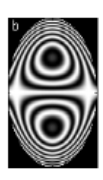

$-1.5 \lambda$

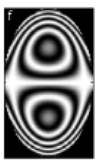

$1.0 \lambda$

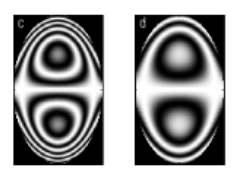

$-0.5 \lambda$

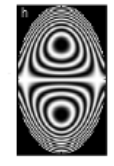

$2 \lambda$
Fig. 3. Interference fringes between a plane wavefront and the aberrated wavefront with the coma1 coefficients from $-2 \lambda$ to $2 \lambda$.

As we can see on Fig. 3, it is difficult to visually distinguish the difference between positive and negative value of the same coefficient (e.g., $-2 \lambda$ and $2 \lambda$ ) in interferometric estimation, whereas our holographic wavefront sensor can easily distinguish between the two, as is shown below Fig. 4.
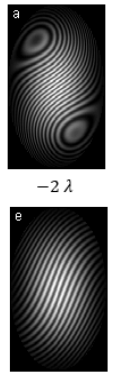

$0.5 \lambda$

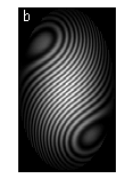

$-1.5 \lambda$

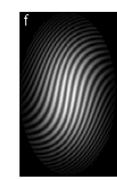

$1.0 \lambda$
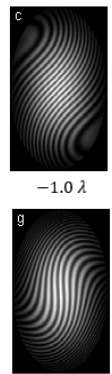

$1.5 \lambda$
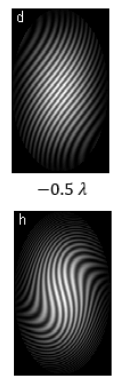

$2 \lambda$

Fig. 4. Interference fringes between the aberrated wavefront and Fourier transform of dot image with the coma1 coefficients from $-2 \lambda$ to $2 \lambda$.

These holograms are added to get the multiplexed hologram, which is then binarized to get the CGH mask. This is shown in Fig. 5.

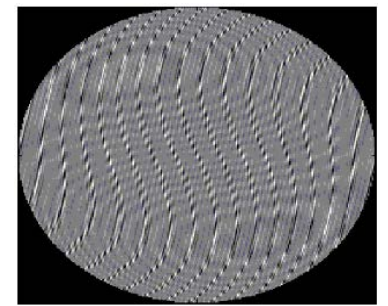

Fig. 5. Binary mask for coma1, coded with amplitudes of Fig. 4.
To check the wavefront sensing for coma1 with this CGH, we use the same aberrated wavefronts one by one as reference and compute the spot images at Fourier plane. The results are shown in Fig. 6. The dots corresponding to the amplitudes are visible clearly.
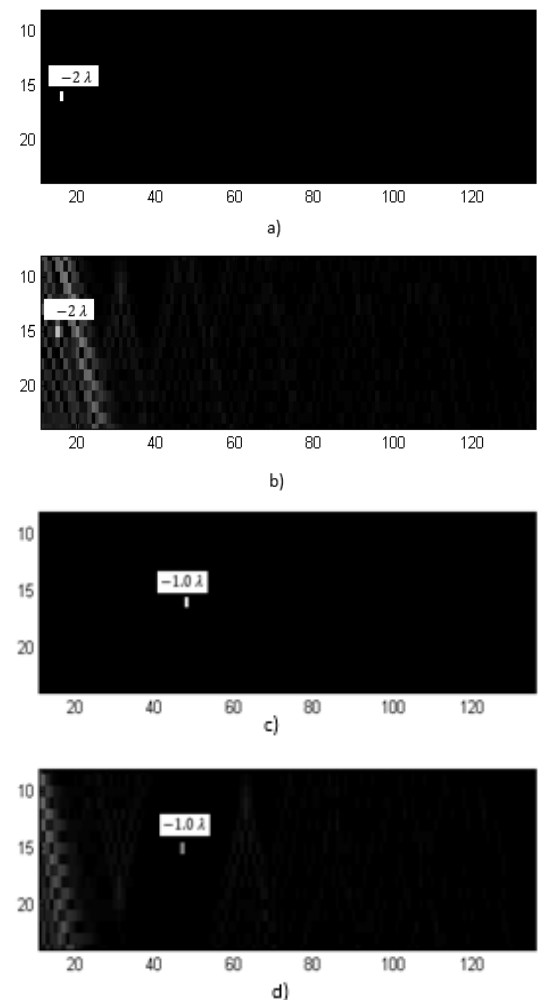

Fig. 6. Sensor output for detection of coma 1 with amplitude $-2 \lambda$ and $1.0 \lambda$, which is coded in the design of CGH mask of Fig. 5. (a, c) original dot images, (b, d) reconstructed dot images.
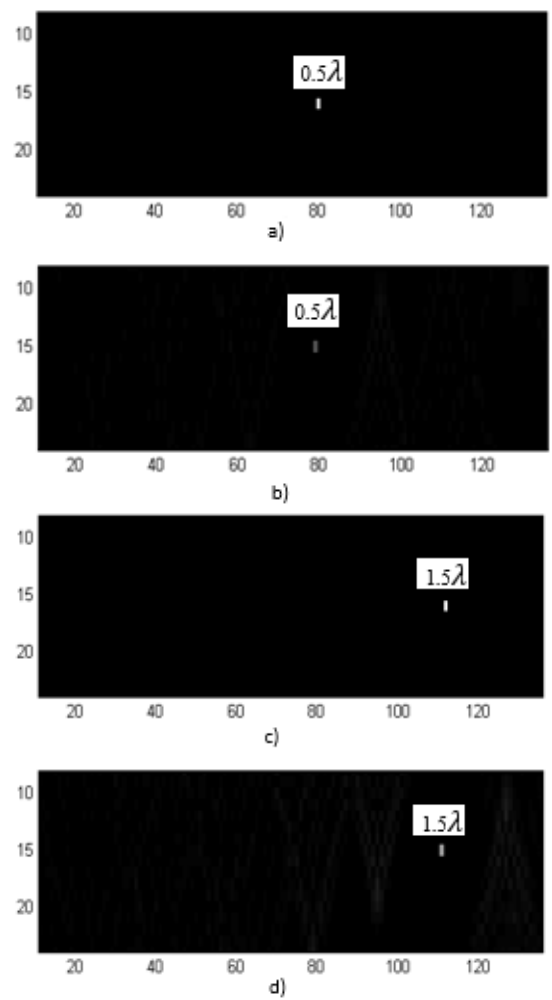

Fig. 7. Sensor output for detection of coma 1 with amplitude $0.5 \lambda$ and $1.5 \lambda$, which is coded in the design of CGH mask of Fig. $5 .(a, b)$ original dot images, (c, d) reconstructed dot images. 
If we illuminate this hologram with a reference wave $\Psi_{R}^{k}$ we get the corresponding dot image dp in the Fourier plane, provided that the hologram of reference wavefront $\Psi_{R}^{k}$ and FT (dp) is already coded in $\mathrm{H}$.

The position of dot image is $\mathrm{d} 1(15,15)$. Here, $\mathrm{d} 1(15,15)$ means that there is a white pixel at $(15,15)$, whereas the rest of the pixels are black.

We take $x=15$ for coma1, so that signal on the line $x=15$ will represent the presence of this mode only.

As shown in Fig. 6, the position of dot image reconstruction from multiplexed hologram is $\mathrm{d} 3(15,48)$. It represents the existence of the aberration coma 1 with amplitude $-1.0 \lambda$.

The corresponding sensor outputs, with 0.5 and 1.5 amplitudes (in $\lambda$ ), are shown in Fig. 7.
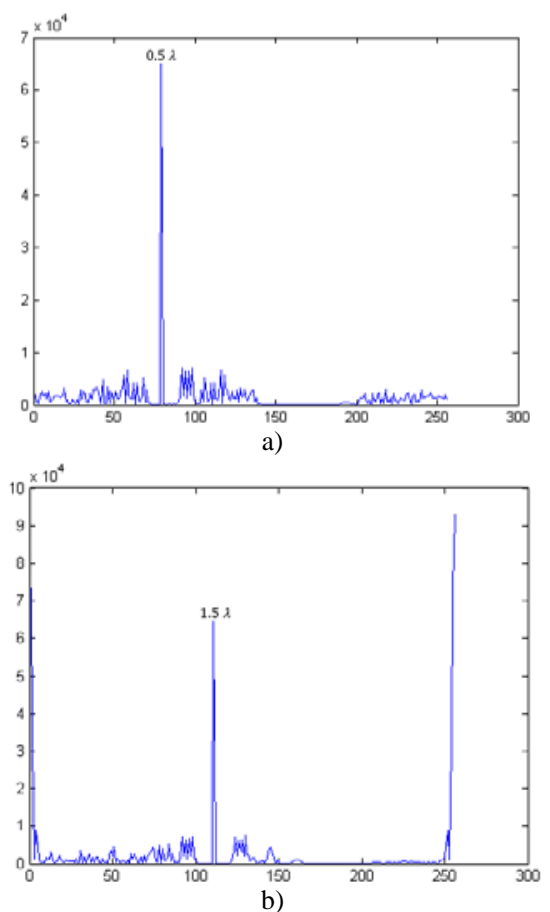

Fig. 8. Plots of sensor outputs, which are coded in the design of CGH mask of Fig. 5. a) detection of coma 1 amplitude $0.5 \lambda$, b) detection of coma1 amplitude $1.5 \lambda$.

As shown in Fig. 8, for the amplitude value, which is coded in the hologram, the sensor gives the signal at the coded value.

For the amplitude value, which is not coded in the hologram, the sensor gives the signal at the nearby-coded value. The results with the amplitude 0.6 and 1.75 are shown in Fig. 9 and Fig. 10 respectively.

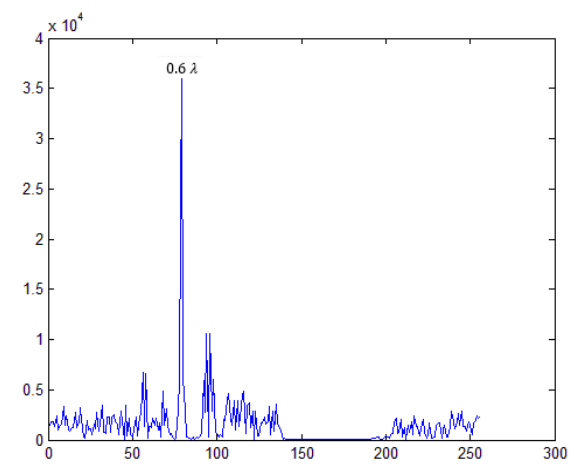

Fig. 9. Plots of sensor output at $x=15$ for detection of coma 1 with amplitude $0.6 \lambda$, which is not coded in the design of CGH mask of Fig. 5.
Fig. 9 shows that for 0.6 (coma1 in $\lambda$ ) amplitude in the input beam, the sensor gives the spot at its nearest-coded value, i.e., 0.5 .

Fig. 10 shows that for 1.25 and 1.75 (coma1 in $\lambda$ ) amplitudes which are not coded in the design of CGH mask of Fig. 5, the sensor gives two feeble spots at equidistant nearest-coded values, i.e., at 1.0 and 1.5 for $1.25 \lambda$ and 1.5 and 2.0 , for $1.25 \lambda$.
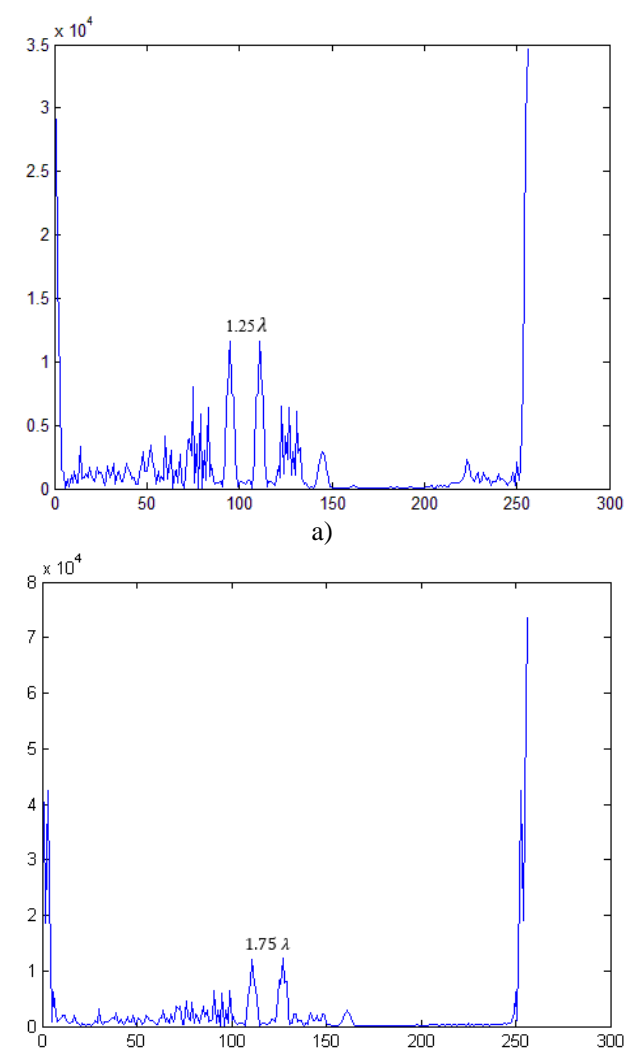

b)

Fig. 10. Plots of sensor outputs, which are not coded in the design of CGH mask of Fig. 5. a) detection of coma1 amplitude $1.25 \lambda$, b) detection of coma1 amplitude $1.75 \lambda$.

\section{CONCLUSION}

In this paper, we simply introduced the basic principle of $\mathrm{CGH}$, which acts as the wavefront sensor. Interference between the aberrated wavefront with a single modecoefficient combination and the Fourier transform of a single dot is numerically calculated for one hologram. Different coefficients for a single mode (coma1) and corresponding different positions of spots are taken to compute various holograms and then all the holograms are multiplexed to get the CGH mask. There is a clear visual distinction between negative and positive value of the same amplitude (e.g., -0.5 and 0.5) in holographic sensor, whereas in the interferometric estimation Fig. 3, the visual result is same for both signs of same value.

\section{REFERENCES}

[1] R. J. Noll, "Zernike polynomials and atmospheric turbulence," Journal of Opt. Soc. Am., vol. 66, no. 3, pp. 207-211, March 1976.

[2] J. M. Geary, Introduction to Wavefront Sensors, USA: SPIE Optical Engineering Press, 1995, ch. 4, pp. 53-64.

[3] K. L. Baker and M. M. Moallem, "Iteratively weighted centroiding for Shack-Hartmann wavefront sensors," Opt Exp, vol. 15, no. 8, pp. 5147-5159, 2007. 
[4] P. M. Blanchard, D. J. Fisher, S. C. Woods, and A. H. Greenaway, "Phase diversity wave-front sensing with a diffraction grating," Appl Opt, vol. 39, no. 35, pp. 6649-6655, 2000.

[5] P. Dyrud and G. Andersen, "Fast holographic wavefront sensor," in Proc. SPIE, 2006, vol. 6215.

[6] F. Ghebremichael, G. P. Andersen, and K. S. Gurley, "Holographybased wavefront sensing,” Appl Opt; vol. 47, no. 4, pp. 62-69, 2008.

[7] R. Bhatt, S. K. Mishra, D. Mohan, and A. K. Gupta, "Direc amplitude detection of Zernike modes by computer-generated holographic wavefront sensor: Modeling and simulation,” Optics and Lasers in Engineering, vol. 46, pp. 428-439, June 2008.

[8] L. Changhai, Y. Yang, S. Guo, R. Xu, T. Men, and C. Wen, "Modal wavefront sensor employing stratified computer-generated holographic elements,” Optics and Lasers in Engineering, vol. 51, no. 11, pp. 1265-1271, 2013.

[9] J. W. Goodman, Introduction to Fourier Optics, 2nd ed. New York: McGraw-Hill, 1996, pp. 96-120.

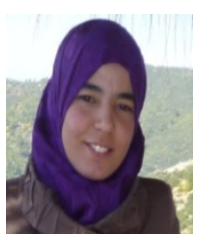

Soumaya Kara Mohammed received her B.S. degree in 2001. She completed her M.Sc. degree in spatia optics and precision mechanics from Ferhat Abbas University of Setif, Algeria in 2010.

At present, she is working as a researcher at the Centre for Development of Advanced Technologies (CDTA), Research Unit in Optics and Photonics (UROP). She is currently preparing the PhD degree in space optics with the institute of Optics and Precision Mechanics at the same university.
Her research interests include holograms, digital holography, optical image processing.

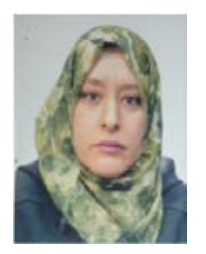

Sabah Cherif received her B.S. degree in 2001. She completed her M.Sc. degree in space Optics and precision mechanics from Ferhat Abbas University of Setif, Algeria in 2010.

At present, she is working as a researcher at the Centre for Development of Advanced Technologies (CDTA), Research Unit in Optics and Photonics (UROP). She is currently preparing the $\mathrm{PhD}$ degree in space Optics with the Institute of Optics and Precision Mechanics at the same university.

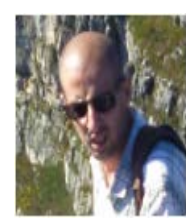

Mahdi Rahmani received his B.S. degree in 2002. He completed his M.Sc. degree in space optics and precision mechanics from Ferhat Abbas University of Setif, Algeria in 2010.

At present, he is working as a researcher at the Centre for Development of Advanced Technologies (CDTA), Research Unit in Optics and Photonics (UROP). He is currently preparing the $\mathrm{PhD}$ degree in space Optics with the Institute of Optics and Precision Mechanics at the same university. 